\section{Tophaceous Pustule-like Rash in a Patient with Gout}

\section{To the Editor:}

Gout is characterized by extracellular fluid urate saturation leading to monosodium urate (MSU) crystal deposition. Typically, it manifests as recurrent acute inflammatory arthritis flares that may lead to a chronic tophaceous destructive arthritis. MSU crystal deposition usually occurs in articular and periarticular areas. MSU crystals are found in cartilage, tendon sheaths, synovial fluid, and subcutaneous tissue ${ }^{1}$. A cutaneous pustulelike rash filled with tophaceous material is a rare presentation of gout. We describe a patient with gouty arthritis presenting with an asymptomatic cutaneous diffuse rash consisting of pustule-like lesions containing tophaceous material.

A 30-year-old man presented with a 6-year history of intermittent polyarticular arthritis involving the knees bilaterally, ankles bilaterally, left elbow, right fifth metacarpophalangeal (MCP) joint, and left olecranon bursitis. In addition, he had a cutaneous diffuse rash. He failed to provide a clear history regarding the time onset of the rash and its course. He did not report any pruritus, tenderness, or pain secondary to his rash. He was diagnosed as having rheumatoid arthritis by his primary doctor and was treated with naproxen, without improvement.

He had no other known medical history. There was no family history of gout or kidney stones. He did not smoke or drink and his only medication was naproxen. When presenting to the rheumatologist's clinic he was found to be obese (body mass index $31 \mathrm{~kg} / \mathrm{m}^{2}$ ). His vital signs were within normal limits. The rest of the examination was unremarkable except for polyarticular synovitis of the knees bilaterally, right fifth MCP joint, and the left elbow and nodular deposits suspected to be tophi. The deposits were in the helix of his right ear, measuring $1.5 \times 0.5 \mathrm{~cm}$; the dorsum of the second and third MCP joint of the right hand, measuring $1 \mathrm{~cm}$; the left olecranon bursa; the right Achilles tendon insertion, measuring $3 \times 2 \mathrm{~cm}$; and bilateral big toes. In addition, he had a rash on his left arm. The rash was filled with white fluid and surrounded by an erythematous base, suggestive of pustules (Figure 1). The size of the lesions was $3-5 \mathrm{~mm}$. No ulcerations were observed. His laboratory investigation was significant for a serum urate level of $10.6 \mathrm{mg} / \mathrm{dl}$ and a glomerular filtration rate $>60 \mathrm{ml} / \mathrm{min}$. His serum glucose was within the normal range $(83 \mathrm{mg} / \mathrm{dl})$. He had hyperlipidemia (total cholesterol $217 \mathrm{mg} / \mathrm{dl}$ and triglyceride $247 \mathrm{mg} / \mathrm{dl}$ ). His sedimentation rate was $30 \mathrm{~mm} / \mathrm{h}$. A urinalysis was normal.

Acute gout and osteomyelitis of the foot can present similarly. The patient had had a recent right toe intraoperative biopsy and culture after being suspected of having osteomyelitis there. The biopsy revealed skin and subcutaneous tissue with multiple gouty tophi. Plain radiographs of the

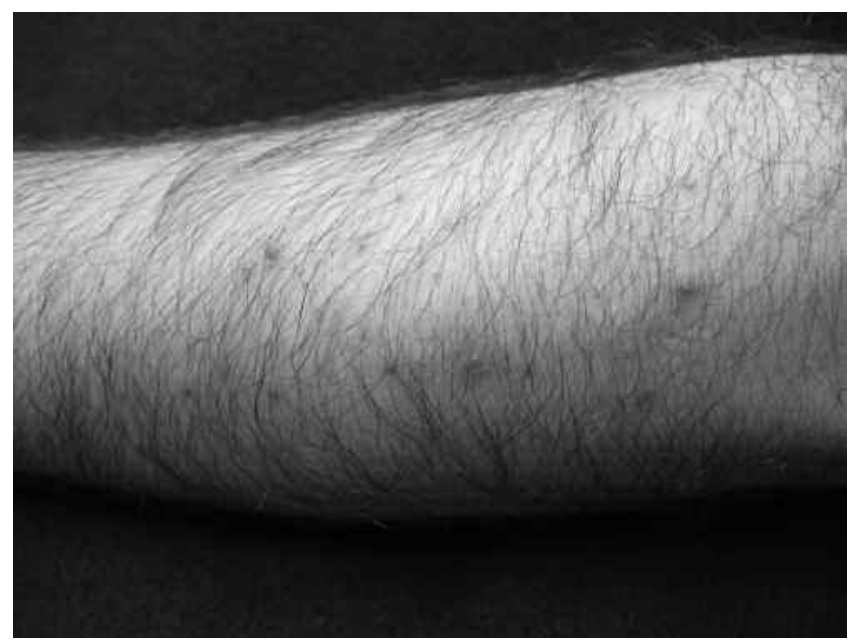

Figure 1. The patient had a rash of tophi filled with white fluid and having erythematous bases, suggestive of pustules.

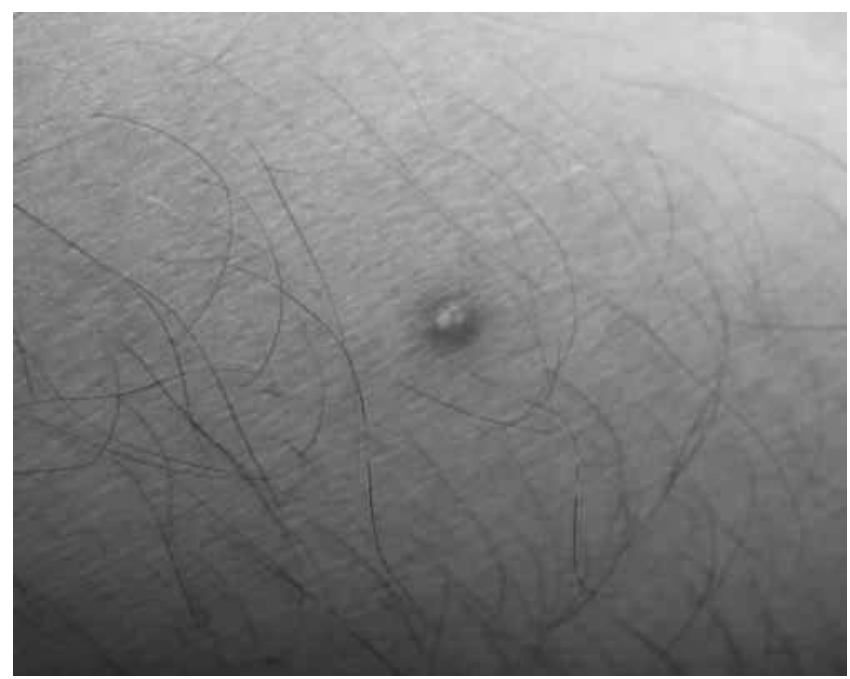

Figure 2. The rash subsided after allopurinol treatment, but large tophi did not seem to shrink.

feet showed erosions in both feet, being most pronounced across the interphalangeal joint of the right great toe. Large soft-tissue masses were observed in the right great toe and the right heel, suggestive of tophi. A plain radiograph of the right hand showed erosions at the bases of the third and fourth middle phalanges and the base of the middle metacarpal, with slight soft-tissue swelling, suggestive of tophaceous material.

Aspiration of one of the skin lesions demonstrated MSU crystals under polarized light microscopy. He was started on a prednisone taper for his acute gout and oral colchicine $0.6 \mathrm{mg}$ daily for flare prevention, as well as allopurinol $200 \mathrm{mg} / \mathrm{day}$ several weeks after his acute attack subsided. One month after starting allopurinol, his serum urate level was down to 8.2 $\mathrm{mg} / \mathrm{dl}$ and gradually his allopurinol dose was increased to $600 \mathrm{mg} /$ day. On an allopurinol dose of $600 \mathrm{mg} /$ day, his recent serum urate was $7.6 \mathrm{mg} / \mathrm{dl}$. After 3 months of allopurinol his skin rash subsided, but his large tophi did not seem to shrink (Figure 2)

Gout can have a wide array of clinical manifestations. The typical ones include acute gouty arthritis attacks, chronic tophaceous destructive gouty arthritis, cutaneous tophi, nephrolithiasis, and nephropathy. Tophi are usually firm nodular subcutaneous deposits, but may rarely be intradermal bullae or plaques.

Tophi presenting as cutaneous bullae or a pustule-like rash have only rarely been reported in the English literature. Schumacher ${ }^{2}$ describes 2 patients with tophi resembling bullae after trauma, one after a burn. Apibal, et $a l^{3}$ described a patient who developed bullae acutely in both her palms and soles; Garcia-Porrua, et $\mathrm{al}^{4}$ reported a young woman with anorexia nervosa who developed palmar bullae initially thought to be pustules; Nogueira, et $a l^{5}$ reported a 56 -year-old woman who presented with a 6-month history of bullae and whitish plaques of the fingers. She had chronic renal failure with no inflammatory arthritis. Khanna and Shrivastava ${ }^{6}$ reported a patient with intradermal bullae on the margins of the sole and heel. Magid, et $a l^{7}$ reported a patient with spinal cord compression secondary to tophaceous deposits who also had pustule-like lesions on his arms, chest, and legs. Fam and Assaad ${ }^{8}$ described a case series of 6 patients with intradermal tophi appearing as small, superficial, pustule-like whitish lesions. Aspiration of the bullae in all the patients described above revealed MSU crystals.

Our patient had a diffuse pustule-like tophaceous rash. Although he was obese, he was young and had no other comorbidities contributing to gout, nor did he have a family history of gout or nephrolithiasis. He had no history of acute or repetitive trauma to the skin, a known precipitating factor for gouty attacks. Shukla, et $a l^{9}$ proposed that risk factors predisposing an individual to development of intradermal gouty tophi include renal 
insufficiency, hypertension, chronic diuretics therapy, long disease duration, and lack of chronic urate-lowering treatment. Possibly our patient developed such disseminated cutaneous tophaceous gout because of his overwhelming uric acid burden. He had no other risk factors.

When a patient presents with synovitis and a diffuse cutaneous pustule-like rash, gout should be included in the differential diagnosis.

MOHAMAD RAFIK CHEHAB, MD; JANAK GOYAL, MD, Department of Medicine, Raritan Bay Medical Center, Perth Amboy, New Jersey; NAOMI SCHLESINGER, MD, Department of Medicine, UMDNJ-Robert Wood Johnson Medical School, Post Office Box 19, New Brunswick, New Jersey 08903, USA. Address correspondence to Dr. Schlesinger; E-mail: schlesna@umdnj.edu

\section{REFERENCES}

1. Sokoloff L. The pathology of gout. Metabolism 1957;6:230-43.

2. Schumacher HR. Bullous tophi in gout. Ann Rheum Dis 1977;36:91-3.
3. Apibal Y, Jirasuthus S, Puavilai S. Abruption pustular gouty tophi of palm and sole. J Med Assoc Thai 2009;92:979-82.

4. Garcia-Porrua C, Canedo C, Argueso R, Gonzalez-Gay MA. Superficial collections of fluid tophaceous material as clinical presentation of anorexia nervosa. Ann Rheum Dis 2005;64:1658-9.

5. Nogueira A, Duarte AF, Morais P, Magina S, Azevedo F. Whitish bullae on the fingers: What is the diagnosis? Tophaceous gout. Dermatol Online J 2009;15:9.

6. Khanna D, Shrivastava A. An unusual presentation of polyarticular tophaceous gout. Rheumatology 2006;45:1095.

7. Magid SK, Gray GE, Anand A. Spinal cord compression by tophi in a patient with chronic polyarthritis. Arthritis Rheum 1981;24:1431-4.

8. Fam AG, Assaad D. Intradermal urate tophi. J Rheumatol 1997;24:1126-31.

9. Shukla R, Vender RB, Alhabeeb A, Salama S, Murphy F. Miliarial gout (a new entity). J Cutan Med Surg 2007;11:31-4.

J Rheumatol 2012;39:1; doi:10.3899/jrheum.110376 